\title{
Prevalence, antibiotic sensitivity pattern and genetic analysis of extended spectrum beta lactamase producing Escherichia coli and Klebsiella spp among patients with community acquired urinary tract infection in Galle district, Sri Lanka
}

\author{
Upul Priyadharshana ${ }^{1}$, Lasanthi B Piyasiri ${ }^{1}$, Champa Wijesinghe ${ }^{2}$ \\ (Index words: extended spectrum beta lactamases, community acquired, urinary tract infection)
}

\begin{abstract}
Introduction Community acquired urinary tract infections (CA-UTI) are commonly caused by Escherichia coli and Klebsiella spp which are known extended spectrum beta lactamase (ESBL) producers.

Objectives To determine, the prevalence and characteristics of ESBL producing of E. coli and Klebsiella spp in the community, and the association of risk factors with ESBL CA-UTI.

Methods Descriptive cross-sectional study with urine cultures performed from clinically suspected CA-UTI patients by CLSI standards. Conventional multiplex PCR was performed for gene analysis.

Results Cultures were positive in $178(38 \%)$ patients from 465 . Majority were females $(103,58 \%)$. Most frequently isolated was E. coli $(149,84 \%)$ with $68(46 \%)$ ESBL producers followed by $16(9 \%)$ Klebsiella pneumoniae with $4(25 \%)$ ESBL producers. Majority of patients with ESBL CA-UTI were $>50$ yrs $(35 / 72,49 \%)$ and $13(18 \%)$ children $<10$ years were present. ABST of ESBL producers revealed high resistance rates for quinolones $(41 \%)$ and $>80 \%$ sensitivity for nitrofurantoin, fosfomycin, mecillinam, aminoglycosides and carbapenems. Presence of ESBL genes were $83 \%$ CTX-M, $71 \%$ OXA, 24\% TEM and 9\% SHV with one organism often producing more than one gene in 29 isolates $(71 \%)$. Haematuria and structural abnormalities of urinary tract were significantly associated with increased ESBL CA-UTI $(p<0.01)$.

Conclusions ESBL prevalence of this community was $40 \%$ in CA-UTI with E.coli predominance among female majority. $>80 \%$ ESBL organisms show high sensitivity for aminoglycosides, carbapenems, nitrofurantoin, mecillinam and fosfomycin. Frequently isolated ESBL
\end{abstract}

gene was CTX-M. Haematuria and structural abnormalities of urinary tract were significantly associated with ESBL CA-UTI.

Ceylon Medical Journal 2019; 64: 140-145

DOI: http://doi.org/10.4038/cmj.v64i4.8990

\section{Introduction}

Extended beta lactamase (ESBL) producing organisms were identified in 1960 due to plasmid mediated antibiotic resistance (CTX-1 gene). Since then several other genes causing ESBL resistance were identified such as CTX-M, SHV, TEM, VEB, and PER. ESBLs render resistance to penicillin, cephalosporins and monobactams. Unlike most of such genes, CTX-M type is known to be linked with multi-drug resistance which includes aminoglycosides, quinolones and cotrimoxazole [1] as well.

ESBLs are basically produced by the enterobacteriaceae family; in particular Klebsiella pneumoniae and Escherichia coli [2]. Other enterobacteriaceae and nonfermenting bacteria like Acinetobacter and Pseudomonas also produce ESBL [3] but prevalence is low. Most of the urinary-tract infections (UTI) are due to Escherichia coli and Klebsiella spp and studying community acquired (CA) UTI is more practical with non-invasive urine cultures comparatively. And these results often reflect antibiotic sensitivity pattern in the community setting.

There had been similar studies in the past. However, many previous studies on risk factors for ESBL were based on the samples received in the routine culture laboratory. Information were often taken from the request forms or bed head tickets are highly unsatisfactory when consi-

${ }^{1}$ Teaching Hospital Karapitiya, Galle, ${ }^{2}$ Faculty of Medicine, University of Ruhuna, Galle, Sri Lanka.

Correspondence: LBP, e-mail: <bhagyapiyasiri@yahoo.co.uk>. Received 06 June 2019 and revised version 18 November 2019 accepted 14 December 2019.

This is an open-access article distributed under the terms of the Creative Commons Attribution License, which permits unrestricted use, distribution, and reproduction in any medium, provided the original author and source are credited. 
dering all potential risk factors for community acquired ESBL infections.

Also, according to Clinical and Laboratory Standards Institute (CLSI 2017 M-1009), ESBL screening in the routine cultures is not recommended, but if done for epidemiological purpose ESBL screening can be done with maximum results with 5 antibiotics such as cefpodoxime $10 \mu \mathrm{g}$, ceftazidime $30 \mu \mathrm{g}$, astreonam $30 \mu \mathrm{g}$, cefotaxime $30 \mu \mathrm{g}$, and ceftriaxone $30 \mu \mathrm{g}$. If the screening is positive, confirmatory tests are carried out but not routinely. As most of the previous studies were based on normal laboratory results there can be some deficiencies in ESBL detection.

Further, in Sri Lanka a lot of patients with CA UTI are treated with empirically at the out-patient department and at general practitioners (GP) and therefore most of these patients were not included in the previous studies as they were not admitted. However, this study was designed to include such patients as well with active sampling and direct interviews with the patient (in both OPD and GP centers) to gather data to see the prevalence of community acquired UTI by ESBL producing coliforms and risk factors associated with the food habits, lifestyle and others.

\section{Objectives}

In this study, we investigated the prevalence of ESBL producing Escherichia coli and Klebsiella species in CA UTI. Antibiotic sensitivity pattern and genetic analysis of ESBL producing bacteria, and the association of risk factors and the clinical presentation with CA ESBL UTI were also described.

\section{Materials and methods}

A descriptive cross-sectional study was carried out for 4 months from December 2016 to March 2017. Study sites were Teaching Hospital Karapitiya (THK) and 10 selected General practitioners' (GP) centers in the Galle district. THK is the $3^{\text {rd }}$ largest tertiary care hospital in Sri Lanka which usually sees about 1500 OPD patients and nearly 1500 newly admitted patients daily.

\section{Study population}

Galle district population in the area surrounding Teaching Hospital Karapitiya, who had CA-UTI during this time period considered as the study population.

\section{Inclusion criteria}

Patients who came to OPD, clinics and GP centers with clinically suspected CA UTI according to the history were included in the study.

\section{Exclusion criteria}

Patients who had hospital admission within the last 3 months, or admitted to the hospital for more than 48 hours or patients on urinary catheters were excluded because we wanted to exclude recurrences due to partially treated urinary tract infections and health care associated infections. Patients who were on antibiotics other than prophylaxis were also excluded.

\section{Sample size}

Sample size was calculated as 173 according to the study by Dissanayake et al, (2010) [4] in which the ESBL prevalence among CA-UTI patients was $13 \%$. For the calculation the standard formula $n=Z^{2} \mathrm{pq} / \mathrm{d}^{2}$ was used with margin of error (d) of $5 \%$.

\section{Data extraction sheet}

By an interviewer based structured questionnaire, information on basic demography, clinical features, about past history of UTI, prophylactic antibiotics, structural anomalies, and about possible risk factors (diabetes, malignancy, chronic renal failure, steroid drugs, visit to India, fresh recreational swimming, consumption of fish / chicken, family history of UTI, health care worker in the immediate family) were gathered. We assumed that an average person in Galle district would consume about $50 \mathrm{~g}$ of chicken per week and about $50 \mathrm{~g}$ of fish 3 times per week according to the household expenditure survey report 2012/13 [5].

\section{Ethical approval}

Ethical approval was obtained from ethics review committees of the Faculty of Medicine, Galle and the Medical Research Institute, Colombo 08.

\section{Methodology}

With the help of medical professionals all clinically suspected CA-UTI patients were identified in the OPD and the GP centers. GP centers were selected according to the consent of the particular GP, facilities available for onsite collection and the distance to the laboratory. After taking the informed written consent, the questionnaires were completed by the principal investigator. Only patients with the history suggestive of CA-UTI were included and sample collection was carried out throughout the intended 4 months to complete the target sample size. Patients were guided on the correct way of collecting urine for culture. One sample was collected from each consented patient into sterile bottles and were immediately transported to the laboratory during day time or kept in the $4^{\circ} \mathrm{C}$ refrigerator if collected after $5 \mathrm{pm}$. Storage time did not exceed 12 hours in any case.

Sample processing was done according to the standard laboratory manual [6]. Urine samples were plated on HiCrome UTI Agar and the plates were read after overnight aerobic incubation at $37^{\circ} \mathrm{C}$. Pure cultures of colony count $>10$ were taken as significant as the samples were taken only from the symptomatic patients. Bacterial 
species were initially identified using Gram stain and basic laboratory tests and the Gram negatives were identified by using REMEL's RapID ${ }^{\mathrm{TM}}$ ONE system to the species level.

Antibiotic sensitivity test (ABST) was done according to CLSI 2017 M-100 (7). All Gram-negative organisms were tested for $1^{\text {st }}$ line antibiotics; ampicillin, cefuroxime, cefotaxime, amoxicillin/clavulanic, gentamicin, ciprofloxacin, co-trimoxazole, norfloxacin, nitrofurantoin, nalidixic acid, and cephalexin.

Also, cefpodoxime $10 \mu \mathrm{g}$, ceftazidime $30 \mu \mathrm{g}$, aztreonam $30 \mu \mathrm{g}$, and ceftriaxone $30 \mu \mathrm{g}$ were added to the primary ABST. If their zone diameters were less or equal to the standards, ESBL confirmatory test was performed for all E.coli and Klebsiella spp.

ESBL confirmatory test was done using double disc method with cefotaxime $30 \mu \mathrm{g}$, cefotaxime /clavulanic acid $30 / 10 \mu \mathrm{g}$, and ceftazidime $30 \mu \mathrm{g}$, ceftazidme /clavulanic 30/ $10 \mu \mathrm{g}$. If the zone diameter deference between combined disc and non-combined disk is $\geq 5 \mathrm{~mm}$, it was confirmed as ESBL producing organism.

These organisms were further tested with amikacin, imipenem, meropenem, and netilmicin. Only Escherichia coli isolates were checked with fosfomycin, and mecillinam. Identified organisms were stored in the nutrient agar broth at $-70^{\circ} \mathrm{C}$ for conventional multiplex PCR later at the Genetech Institute (Colombo) according to the in-house protocols by the principal investigator.

\section{Data analysis}

The comparison of bacteriological profiles was analyzed by Statistical Package for Social Sciences (SPSS) software version 22. To analyze the association between categorical variables Pearson's chi-square test was used.

\section{Results}

During the study period, data and urine samples were collected from 465 of clinically suspected symptomatic CA UTI patients from OPD (240), GP centers (150) and from hospital clinics (75). We continued to collect samples throughout the intended study period despite the calculated sample size being 173 . We had $178(38 \%)$ culture positives and were recruited in the study.

From that collection, 72 (40.4\%) ESBL forming Escherichia coli and Klebsiella pneumoniae, 93 non-ESBL Escherichia coli and Klebsiella pneumoniae and 13 other bacterial species were identified (Table 1).

\section{Table 1. Isolated organisms from culture positive CA UTI patients (Total=178)}

\begin{tabular}{lc}
\hline Isolates & Number \\
\hline Non ESBL Escherichia coli & $81(45.5 \%)$ \\
ESBL Escherichia coli & $68(38.2 \%)$ \\
Non ESBL Klebsiella pneumoniae & $12(6.7 \%)$ \\
ESBL Klebsiella pneumoniae & $4(2.2 \%)$ \\
Enterobacter spp & $2(1.1 \%)$ \\
Enterococcus spp & $6(3.4 \%)$ \\
Proteus spp & $2(1.1 \%)$ \\
Pseudomonas aeruginosa & $2(1.1 \%)$ \\
Coagulase negative Staphylococcus spp & $1(0.6 \%)$ \\
\hline
\end{tabular}

Majority was females among the total positives (103/ $178,58 \%$ ) and among the total ESBL positives (40/72, 56\%) but the association was not statistically significant $(\mathrm{p}=0.637)$. The mean age was 39.58 years with $90 \mathrm{yrs}$ being the maximum and most of ESBL producing organisms were isolated from age between 51-60 years (Figure 1).

Table 2. BST of ESBL E.coli and Klebsiella spp isolates $(\mathrm{n}=72)$

\begin{tabular}{|c|c|c|c|}
\hline Antibiotic & Sensitive & Intermediate & Resistance \\
\hline Gentamicin $10 \mu \mathrm{g}$ & $57(79 \%)$ & 0 & $15(21 \%)$ \\
\hline Ciprofloxacin $5 \mu \mathrm{g}$ & $15(21 \%)$ & 0 & $57(79 \%)$ \\
\hline Co-trimoxazole $1.25 / 23.75 \mu \mathrm{g}$ & $29(40 \%)$ & 0 & $43(60 \%)$ \\
\hline Norfloxacin $10 \mu \mathrm{g}$ & $11(15 \%)$ & 0 & $61(85 \%)$ \\
\hline Nitrofurantoin $300 \mu \mathrm{g}$ & $64(89 \%)$ & $1(1 \%)$ & $7(10 \%)$ \\
\hline Nalidixic acid $30 \mu \mathrm{g}$ & $9(12 \%)$ & 0 & $63(88 \%)$ \\
\hline Amikacin $10 \mu \mathrm{g}$ & $72(100 \%)$ & 0 & 0 \\
\hline Imipenem $10 \mu \mathrm{g}$ & $72(100 \%)$ & 0 & 0 \\
\hline Meropenem $10 \mu \mathrm{g}$ & $70(97 \%)$ & 0 & $2(3 \%)$ \\
\hline Netilmicin $30 \mu \mathrm{g}$ & $68(94 \%)$ & 0 & $4(6 \%)$ \\
\hline Fosfomycin $200 \mu \mathrm{g}$ - only for E.coli & $68(100 \%)$ & 0 & 0 \\
\hline Mecillinam $10 \mu \mathrm{g}$ - only for E.coli & $62(91 \%)$ & $4(6 \%)$ & $2(3 \%)$ \\
\hline
\end{tabular}




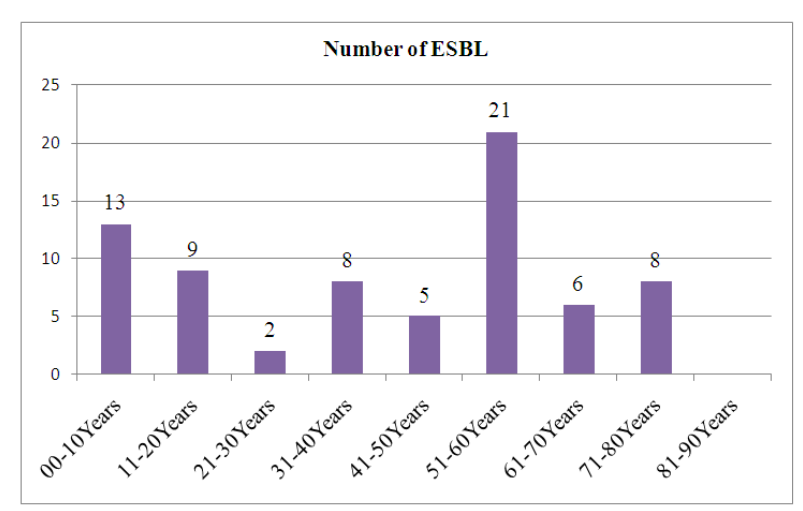

Figure 1. Isolation of ESBL E.coli and Klebsiella spp among age groups.

\section{Antibiotic sensitivity pattern}

Oral antibiotics which are being commonly used empirically in the community set-up were noted to have high resistance rates for E.coli and Klebsiella pneumoniae; co-amoxiclav 59\% (97/165 of total E.coli and Klebsiella), norfloxacin 48.5\% (80/165), co-trimoxazole $43.6 \%$ (72/165), ciprofloxacin $41 \%$ (68/165) and nalidixic acid 28\% (46/165).

All E.coli and Klebsiella were fully sensitive to imipenem and amikacin (100\% sensitivity) while gentamicin and nitrofurantoin had $89.7 \%(148 / 165)$ and $91.5 \%$ (151/165) sensitivity respectively. ESBL E.coli showed
$100 \%$ sensitivity to fosfomycin and $91 \%$ sensitivity to mecillinam.

\section{ESBL gene analysis}

Only 43 samples were tested by conventional PCR due to limited funds. Therefore, only 39 ESBL E.coli isolates (randomly selected) out of 68 totals, and all 4 ESBL Klebsiella isolates were analysed.

According to gene analysis, 83\% CTX-M (34/41), 70.73\% OXA (29/41), 24.4\% TEM (10/41) and 9.8\% SHV $(4 / 41)$ were found. PCR was not successful in two E.coli samples.

Out of total ESBL Klebsiella pneumoniae, TEM in $25 \%$, SHV in $75 \%$, OXA in $75 \%$ and CTX-M in $75 \%$ were identified. ESBL E.coli genes were identified as; SHV in $2.7 \%$ (1/37), TEM in $24.32 \%$ (9/37), OXA in 70\% (26/37) and CTX-M in $83.8 \%$ (31/37). More than one gene was identified in 64.86\% (24/37) ESBL E.coli and 75\% (3/4) ESBL Klebsiella pneumoniae. One E.coli isolate and one Klebsiella isolate had all 4 genes in each and showed similar antibiotic sensitivity pattern.

\section{Clinical features}

Statistical significance was analysed for features of UTI such as fever, dysuria, frequency, abdominal pain, renal angle tenderness, haematuria and anuria. There was significant association between haematuria and ESBL CA-UTI.

Table 3. Significance of clinical features and ESBL CA UTI (ESBL=72, non-ESBL=106)

\begin{tabular}{|c|c|c|c|c|c|}
\hline \multirow[t]{2}{*}{ Symptoms/Signs } & \multicolumn{2}{|c|}{ ESBL UTI } & \multicolumn{2}{|c|}{ Non-ESBL UTI } & \multirow[t]{2}{*}{$P$ value } \\
\hline & Positive & Negative & Positive & Negative & \\
\hline Fever & 54 & 18 & 71 & 35 & 0.251 \\
\hline Dysuria & 59 & 13 & 87 & 19 & 0.928 \\
\hline Frequency & 59 & 13 & 78 & 28 & 0.194 \\
\hline Abdominal pain & 45 & 27 & 68 & 38 & 0.822 \\
\hline Renal-angle tenderness & 13 & 59 & 26 & 80 & 0.306 \\
\hline Hematuria & 15 & 57 & 12 & 94 & 0.032 \\
\hline Anuria & 2 & 70 & 6 & 100 & 0.362 \\
\hline
\end{tabular}

\section{Risk factors for ESBL CA-UTI}

Among the risk factors we analysed, only structural abnormalities of the urinary tract was significantly associated with ESBLUTI in the community. 
Table 4. Significance of risk factors and ESBL CA UTI (ESBL-72, non-ESBL=106)

\begin{tabular}{|c|c|c|c|c|c|}
\hline \multirow[t]{2}{*}{ Symptoms/Signs } & \multicolumn{2}{|c|}{ ESBL UTI } & \multicolumn{2}{|c|}{ Non-ESBL UTI } & \multirow[t]{2}{*}{$P$ value } \\
\hline & Positive & Negative & Positive & Negative & \\
\hline Above 65 years & 13 & 59 & 19 & 87 & 0.982 \\
\hline Diabetes & 16 & 56 & 16 & 90 & 0.224 \\
\hline Structural abnormalities of the urinary tract & 8 & 64 & 2 & 104 & 0.022 \\
\hline Visit to India & 1 & 71 & 1 & 105 & 1.000 \\
\hline Steroid use & 0 & 72 & 1 & 105 & 0.654 \\
\hline Fresh-water recreational activities & 2 & 70 & 1 & 105 & 0.734 \\
\hline Chicken consumption (at least $50 \mathrm{~g}$ weekly) & 43 & 29 & 68 & 38 & 0.637 \\
\hline Fish consumption (50g 3 times per week) & 62 & 10 & 82 & 24 & 0.260 \\
\hline Family history of other infections & 0 & 72 & 0 & 106 & - \\
\hline Health care worker in the immediate family & 4 & 68 & 6 & 100 & 1.000 \\
\hline Chronic renal failure & 0 & 72 & 0 & 106 & - \\
\hline Malignancy & 0 & 72 & 0 & 106 & - \\
\hline Past history of UTI & 22 & 50 & 22 & 84 & 0.137 \\
\hline Antibiotic prophylaxis & 0 & 72 & 0 & 106 & - \\
\hline
\end{tabular}

\section{Discussion}

Community acquired urinary tract infection is one of the major reasons to attend to the medical practitioners. Due to antibiotics resistance of major causative organisms of UTI the antibiotic options in the community has been rendered limited.

Only $39.03 \%$ of clinically suspected CA-UTI urine samples yielded positive cultures with Escherichia coli $(83 \%)$ as the main pathogen. Majority of culture positive patients were females $(57.86 \%)$. These facts are compatible with most of previous CA-UTI studies [4].

In this community, ESBL producing Escherichia coli and Klebsiella pneumoniae prevalence was $40.44 \%$ while Mohomed et al in India [8] found almost same in 2007. However, according to the local study done by Dissanayake et al in 2010 [4], the ESBL prevalence found among the CA-UTI patients was $13 \%$.

We noted that, many primary antibiotics such as coamoxiclav (59\% resistance), norfloxacin $(48.5 \%)$ and cotrimoxazole (43.6\%) were shown resistant for E.coli and Klebsiella spp. According to the standard guidelines, to use an antibiotic as an empirical antibiotic it must have been tested $>90 \%$ sensitive on the causative E. coli of that community [9]. According to that criterion, only mecillinam, nitrofurantoin and fosfomycin can be used as oral empirical antibiotics in this community. However, fosfomycin is currently unavailable in Sri Lanka.

When comparing the current study and the study by
Dissanayake et al [4], while imipenem and amikacin sensitivity has been preserved same, nitrofurantoin and ciprofloxacin sensitivity has decreased by $7 \%$ and $24 \%$ respectively during the 8 years gap. No previous studies had tested fosfomycin or mecillinam in Sri Lanka.

It had been identified that most of ESBL producing organisms are having CTX-M gene. We found CTX-M gene on $83 \%$ of samples while OXA (70.73\%), TEM $(24.4 \%)$ and SHV (9.8\%) were also detected. High prevalence of CTX-M can explain the high rates of multiresistance to antibiotics [1].

According to the clinical features we analysed, statistically significant relationship was noted only between haematuria and ESBLCA-UTI. However, several studies have concluded that the clinical picture of UTI cannot exactly predict whether it is ESBL UTI or not [10].

Among risk factors, only structural abnormality of urinary tract was statistically significant in the association with ESBL UTI. In 2013 Søraas et al [11] found several independent risk factors increased the probability of ESBL UTI, namely, travel to Asia, Middle East or Africa either during the past six weeks to 2 years, recent use of fluoroquinolones or beta lactam antibiotics, diabetes mellitus, and freshwater swimming in the past year. They further concluded that increasing number of fish meals will reduce the risk of ESBL producing UTI. In the present study we could not find statistically significant relationship between those same risk factors and some others and ESBL UTI. 


\section{Conclusions}

ESBL prevalence in CA-UTI in this community is $40.44 \%$. Only nitrofurantoin, mecillinam and fosfomycin can be used as empirical oral antibiotics according to sensitivity rates. Most prevalent ESBL gene in this community is CTX-M gene. Haematuria and structural abnormalities of the urinary tract are having statistically significant relationship with ESBL CA-UTI.

\section{Acknowledgements}

We acknowledge the funding from the Medical Research Institute, work of the reviewer Dr S.Chandrasiri, support given by all medical officers in the out-patient department of THK, and in the GP centers, the laboratory staff of THK, ethical and scientific committees of Medical Research Institute, Borella, and the patients.

\section{References}

1. Martinez P, Garzón D, Mattar, D. CTX-M-producing Escherichia coli and Klebsiella pneumoniae isolated from community-acquired urinary tract infections in Valledupar, Colombia. The Brazilian Journal of Infectious Diseases, 2012; 16(5): 420-425

2. Paterson DL, Bonoma RA. Extended-spectrum $\beta$ lactamases: Aclinical update, Clinical Microbiology Reviews 2005; 18: 657-86.

3. Jacoby GA, Munoz-Price LS. The new beta-lactamases, The New England Journal of Medicine 2005; 352(4): 380-91.

4. Dissanayake DMBT, Fernando SSN, Chandrasiri NS. The distribution and characteristics of Extended-Spectrum $\beta$ Lactamase (ESBL) producing Escherichia coli and Klebsiella species among urinary isolates in a tertiary care hospital, Sri Lanka. Journal of Infectious Diseases, 2012; 2(2): 30-6.

5. Household income and expenditure survey 2012/13 final report, Department of Census and Statistics, Sri Lanka: 29.

6. Karunaratne K, Dassanayake M, Wijesuriya T, Nanayakkara K, eds. Laboratory manual in microbiology: The Sri Lanka College of Microbiologists 2011, 2nd ed.

7. Performance Standards for Antimicrobial Susceptibility Testing; Twenty-Fourth Informational Supplement M100S27. Clinical and Laboratory Standards Institute, Wayne, USA, 2017.

8. Mohammed A, Mohammed S, Asad UK. Etiology and antibiotic resistance patterns of community-acquired urinary tract infections in J N M C Hospital Aligarh, India. Annals of Clinical Microbiology and Antimicrobials 2007; 6: 4.

9. Gupta K, Hooton TM, Naber KG, Wullt B, Colgan R, et al. International Clinical Practice Guidelines for the Treatment of Acute Uncomplicated Cystitis and Pyelonephritis in Women: A 2010 Update by the Infectious Diseases Society of America and the European Society for Microbiology and Infectious Diseases. Clinical Infectious Diseases 2011; 52(5): e103-e120.

10. Minejima E, Beringer AW. Differences in Clinical Presentation and Outcome between Extended-Spectrum Beta-Lactamase (ESBL) Versus Non-ESBL Urinary Tract Infections (UTIs) in a Medically Underserved Population, Open Forum Infectious Diseases 2016; 3(suppl): 1.

11. Soraas A, et al. Risk Factors for Community-Acquired Urinary Tract Infections Caused by ESBL-Producing Enterobacteriaceae - A Case-Control Study in a Low Prevalence Country. PLoS ONE, 2013; 8(7): e6958

12. Tilekaratne GL, et al. Extended-spectrum B-Lactamaseproducing Enterobacteriaceae as a Common Cause of Urinary Tract Infections in Sri Lanka. Journal of Infection and Chemotherapy 2016; 48: 160-5. 